\title{
EXERCÍCIOS ENVOLVENDO A MAGNITUDE LIMITE NO ENSINO DE ASTRONOMIA
}

\author{
Lev Vertchenko* \\ Tomás de Aquino Silveira**
}

RESUMO: Neste trabalho são apresentados alguns exercícios e atividades, elaborados para disciplinas de Astronomia Básica e inspirados na proposta de exploração multicontextual de Ausubel, relacionando o conceito de magnitude limite ao poder de captação de luz de um instrumento ótico. Os resultados desses exercícios são comparados a contagens de estrelas, obtidas seja pela observação, a olho nu ou com instrumentos óticos, seja pelo uso de software do tipo carta celeste. O processo inclui a familiarização do estudante com o céu, com binóculos e telescópios e com vários softwares. São discutidas a relação dessas contagens com a distribuição espacial das estrelas, e sua aplicabilidade a situações de ensino/aprendizagem. Palavras-chave: Astronomia; Ensino; Magnitude.

\section{EXERCISES COMPREHENDING THE LIMIT MAGNITUDE IN ASTRONOMY TEACHING}

ABSTRACT: We present a set of exercises and activities, designed for Basic Astronomy disciplines and inspired by Ausubel's multi-contextual approach, relating the limit magnitude concept to the light catching power of an optical instrument. These exercises are compared with stars count , through naked-eye observations, or those made with the aid of optical instruments, and through the use of star charts software. Meanwhile, the student has the opportunity to become familiarized with the sky, binoculars and telescopes, as well as with several types of software. It is also discussed the relation of the stars count with the spatial distribution of these objects and their application in teaching/learning circumstances.

Keywords: Astronomy; Teaching; Magnitude.

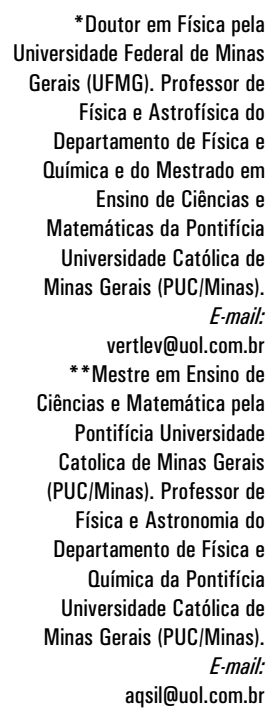

*Doutor em Física pela Universidade Federal de Minas Gerais (UFMG). Professor de

Física e Astrofísica do Departamento de Física e Química e do Mestrado em Ensino de Ciências e Matemáticas da Pontifícia Universidade Católica de Minas Gerais (PUC/Minas). E-mail:

vertlev@uol.com.br * * Mestre em Ensino de Ciências e Matemática pela Pontifícia Universidade Catolica de Minas Gerais (PUC/Minas). Professor de Física e Astronomia do Departamento de Física e Química da Pontifícia Universidade Católica de Minas Gerais (PUC/Minas). E-mail: aqsil@uol.com.br 


\section{Introdução}

Uma grande vantagem da abordagem de tópicos de Astronomia e Astrofísica na formação de profissionais de Física é a frequente integração de conteúdos fascinantes, tendo a observação do Cosmos por contexto. Por exemplo, Bernardes et al. [1] mostram como conteúdos de Ótica podem ser explorados na construção de telescópios. Neste trabalho estendemos a integração de conteúdos de Ótica e Astronomia ao abordarmos exercícios que envolvem um parâmetro fundamental na caracterização observacional dos astros, a magnitude aparente. Mais precisamente, nos concentraremos na magnitude limite, que caracteriza os objetos de brilho mais fraco passíveis de serem observados, e está relacionada ao "poder de captação de luz" do instrumento ótico usado na observação. $\mathrm{O}$ interesse nos exercícios que propomos é ressaltado pelo fato de eles poderem ser acompanhados de tarefas observacionais que podem ser executadas sem maiores dificuldades, dispondo-se de máquina fotográfica, binóculo e software de carta celeste com filtro de magnitude. Atualmente esse tipo de software é muito popular, proporcionando ao seu usuário facilidade na localização e identificação de objetos celestes. A esmerada apresentação gráfica de alguns desses softwares permite, inclusive, que eles sejam usados na simulação da aparência do céu em planetários. Todavia, a aparência do céu proporcionada por tais aplicativos depende criticamente do parâmetro magnitude limite ajustado em seus filtros de magnitude. Será explorada, também, uma ligação com a Cosmologia, pois um importante teste da homogeneidade do Universo, afirmada no chamado "Princípio Cosmológico", envolve a magnitude limite. Este trabalho destina-se a complementar disciplinas de introdução à Astronomia no ensino superior, tanto na formação de professores, como na formação de pesquisadores.

$\mathrm{Na}$ próxima Seção discorreremos brevemente sobre o conceito de magnitude. $\mathrm{Na}$ Seção 3 apresentaremos a fundamentação teórico-pedagógica do trabalho. A Seção 4 constitui o cerne do presente trabalho, onde são apresentados, em sequência adequada, os enunciados dos exercícios e das tarefas observacionais e de manuseio de software. $\mathrm{Na}$ Seção 5 apresentaremos a solução dos exercícios e exemplos de execução das tarefas observacionais e de uso de software. $\mathrm{Na}$ Seção 6, faremos uma discussão final.

\section{Magnitudes astronômicas e poder de captação de luz}

Há mais de dois mil anos o astrônomo grego Hiparco elaborou um sistema para classificação do brilho das estrelas baseado em "magnitudes", atribuindo às estrelas mais brilhantes a magnitude 1 e às estrelas menos brilhantes que, à época podiam ser vistas, obviamente a olho nu, a magnitude 6. Em 1856 este sistema foi formalizado por Pogson [2]. Ele partiu da constatação de que, para se ter uma sensação igual à provocada por uma estrela de magnitude 1, 
seriam necessárias 2,5 estrelas de magnitude 2, e, de modo análogo, 2,5 estrelas de magnitude 3 seriam equivalentes, em brilho, a uma estrela de magnitude 2 . Isso prosseguia até a magnitude 6 , de modo que 2,55 estrelas de magnitude 6 dariam uma sensação de brilho similar ao brilho de uma estrela de magnitude 1. Como $2,5^{5}$ é um número próximo de cem, ele adotou, por definição, que, se as magnitudes de duas estrelas diferem de cinco unidades, então a razão entre seus brilhos é cem³. Assim, a diferença de uma magnitude corresponde, pela definição de Pogson, a , ou seja, aproximadamente 2,512, valor conhecido como razão de Pogson. Chamando os brilhos aparentes de duas estrelas 1 e 2 de, respectivamente, $f_{1}$ e $f_{2}$, e suas magnitudes aparentes correspondentes de $m 1$ e $m 2$, o raciocínio acima pode ser representado pela expressão

$$
\frac{f_{1}}{f_{2}}=(\sqrt[5]{100})^{m_{2}-m_{1}}=100^{\left(m_{2}-m_{1}\right) / 5}, \text { ou } \frac{f_{1}}{f_{2}}=2,512^{m_{2}-m_{1}} .
$$

Se aplicarmos o logaritmo de base 10 aos dois membros da primeira expressão, após um mínimo de trabalho algébrico encontraremos

$$
m_{2}-m_{1}=2,5 \log _{10}\left(\frac{f_{1}}{f_{2}}\right) \text {. }
$$

O caráter logarítmico da relação entre a magnitude e o brilho aparente deve-se a uma lei mais geral da psicofísica, que relaciona os estímulos e respostas percebidas pela chamada Lei de Weber-Fechner' ${ }^{1}$.

Para um observador na Terra, todas as estrelas, excetuando-se o Sol, podem ser consideradas fontes de luz puntiformes, emitindo por igual em todas as direções. Neste caso, o brilho (ou fluxo) aparente caracteriza a diluição espacial da energia luminosa emitida pela estrela quando chega ao observador, sendo definido por

$$
f=\frac{L}{4 \pi d^{2}},
$$

onde $L$ é a luminosidade (ou potência luminosa) da estrela e $d$ é a sua distância à Terra. No caso de observar-se a luz emitida em todos os comprimentos de onda, a luminosidade e a respectiva magnitude chamam-se "bolométricas". Para caracterizar o objeto por uma magnitude que independa da sua distância ao observador usa-se a "magnitude absoluta", de valor igual ao da magnitude aparente do objeto se ele estivesse a uma distância de 10 parsecs do observador.

Todo instrumento ótico possui um limite inferior de detecção do brilho, correspondendo aos objetos mais fracos que podem ser observados por meio dele. O brilho destes objetos no limiar de detecção é chamado de "fluxo limite" e a correspondente magnitude aparente de "magnitude limite". 
Para o olho humano, em condições ótimas de observação, a magnitude limite está próxima de 6 (lembremos que 6 era o valor atribuído por Hiparco à magnitude das estrelas de brilho mais fraco observadas). Este limiar de detecção é determinado pela energia luminosa captada pelo instrumento e pela sensibilidade do sensor ao receber esta energia. Como a energia captada pelo instrumento é condicionada pela sua abertura, o parâmetro área da abertura passa a se chamar "poder de captação de luz". Por exemplo, para o olho o poder de captação é a área da pupila, para um telescópio refletor é a área do seu espelho primário. Vemos, então, que a grande vantagem de se usar telescópio com grande espelho primário é o seu grande poder de captação de luz, permitindo observar objetos mais fracos, de maior magnitude limite. Outra importante vantagem em se ter telescópio com espelho grande é a sua maior capacidade em resolver objetos (a resolução dos objetos observados é dificultada pelo efeito da difração), sendo o "poder de resolução" descrito pelo critério de Rayleigh. No entanto, no presente trabalho vamos nos ocupar apenas do parâmetro "poder de captação de luz". Também consideraremos as magnitudes como bolométricas, por simplicidade, tratando-se de um estudo introdutório, de familiarização com o conceito de magnitude.

\section{Fundamentação teórico-pedagógica}

Não estamos propondo uma metodologia baseada na aprendizagem por descoberta ou construtivista, e sim uma metodologia mais próxima da proposta por Ausubel na exploração multicontextual de exercícios [4]. Em nossa prática, percebemos que os conteúdos de Astronomia necessários à correta utilização da carta celeste geralmente são ensinados por meio da forma que Ausubel chama de "aprendizagem mecânica", sem a preocupação de torná-los significativos no momento da aprendizagem. A essência da teoria da "aprendizagem significativa" de Ausubel encontra-se na ancoragem dos novos conceitos assimilados na estrutura cognitiva do aprendiz. Materiais que facilitariam tal ancoragem são chamados de "organizadores prévios". Assim, a exploração multicontextual dos exercícios propostos pode ser entendida como facilitadora da ancoragem dos novos conceitos de Astronomia, e a sequência de exercícios, dentro deste espírito, pode ser considerada como constituindo organizadores prévios. No caso, o aluno explorará os conceitos de captação de luz e de magnitude, e no processo se familiarizará com a manipulação de softwares de cartas celestes, interpretação de resultados, busca de hipóteses que expliquem discrepâncias com previsões teóricas etc., ou seja, ele estará às voltas com uma verdadeira investigação científica. Para o autor mencionado, algumas práticas expositivas mal sucedidas são: 
1. Uso prematuro de técnicas verbais puras com alunos imaturos em termos cognitivos.

2. Apresentação arbitrária de fatos não relacionados sem quaisquer princípios de organização ou de explicação.

3. Não integração de novas tarefas de aprendizagem com materiais anteriormente apresentados.

4. Utilização de procedimentos de avaliação que avaliam somente a capacidade de se reconhecerem fatos discretos, ou de se reproduzirem ideias pelas mesmas palavras ou no contexto idêntico ao encontrado originalmente (AUSUBEL, 2003, p. 7).

Este trabalho procura caminhar em direção contrária a alguns desses defeitos que Ausubel detecta no ensino, tais como a apresentação arbitrária de fatos não relacionados, "sem quaisquer princípios de organização ou de explicação". Há uma linha, com aumento de complexidade, e com forte contextualização na observação astronômica e no apoio de softwares. Da forma como está proposto, julgamos que este trabalho é adequado para disciplinas de introdução à Astronomia, seja na formação de professores, ou na de pesquisadores.

\section{4 - Exercícios e atividades}

A presente sequência de atividades intercala a resolução "teórica" de exercícios com atividades observacionais a olho nu, ou com o emprego de luneta ou binóculo, e o manuseio de software do tipo carta celeste. É necessário que o software disponha de um filtro de magnitudes, isto é, que permita condicionar a apresentação das estrelas na tela e elas estarem com magnitude aparente inferior à magnitude limite determinada pelo usuário. Por exemplo, podem ser usados os softwares EZCosmos (obsoleto em alguns aspectos, escrito para o sistema operacional DOS, mas com a vantagem da simplicidade ${ }^{2}$ ), Cartes $d u$ Ciel (gratuito, disponível na Internet ${ }^{3}$ ), Stellarium ${ }^{4}$ (também gratuito e disponível na Internet). Existem também softwares que podem ser adquiridos, com recursos mais avançados, como é o caso do Redshift, que já se encontra em sua versão sete ${ }^{5}$. E há outros, como o CyberSky, também disponível na internet ${ }^{6}$, mas cujo uso gratuito está restrito a um certo número de sessões.

\section{$1^{a}$ atividade: manuseio do software}

Faça o software de carta celeste apresentar uma imagem do céu noturno. Localize o seu filtro de magnitudes e anote o valor da magnitude limite usado no momento. Diminua em três unidades a magnitude limite e observe a nova apresentação do céu. Repita o procedimento, agora aumentando três unidades na magnitude limite inicial. Interprete os resultados.

\section{$2^{a}$ atividade: observação a olho nu e manuseio do software}

Observe, a olho nu, uma seção do céu noturno que permita contar o número de estrelas observadas. Conte o número de estrelas observadas na seção do céu escolhida. Localize no software de carta celeste a seção do céu observada. 
Ajuste a magnitude limite do software até que o número de estrelas apresentadas na seção seja igual ao que foi contado na observação. A magnitude ajustada é a magnitude limite do olho do observador.

\section{$3^{a}$ atividade: fotografia do céu noturno e manuseio do software}

Obtenha uma fotografia de uma seção do céu noturno. Localize esta seção no software de carta celeste e obtenha a magnitude limite da foto. Para isto ajuste a magnitude limite no filtro de magnitude do software até que na seção correspondente à foto apareça o mesmo número de estrelas que na foto.

\section{$4^{a}$ atividade: exercício teórico}

Dois fotossensores idênticos são usados na observação de estrelas por meio de dois instrumentos, por exemplo, dois telescópios refletores. O primeiro instrumento possui abertura de diâmetro Do (no caso do telescópio refletor, é o diâmetro do seu espelho primário) e o sensor acoplado a ele detecta estrelas até a magnitude limite mlim $=$ mo. Se o segundo instrumento possui abertura de diâmetro $\alpha \mathrm{D}_{0}$, qual será a magnitude limite (expressa em termos dos parâmetros mencionados) detectada por meio deste telescópio?

Considere que a resposta dos sensores é proporcional ao número de fótons captados pelo espelho principal em um certo tempo, isto é, a resposta é $i \propto$ (área do espelho x fluxo).

$5^{a}$ atividade: caracterização do instrumento de observação

Considere como o primeiro instrumento de observação o seu olho, cuja abertura é dada pelo diâmetro da pupila. Estime o diâmetro da pupila. Considere como segundo instrumento um binóculo ou luneta disponível e que será usado em tarefa adiante. Meça o diâmetro da objetiva deste binóculo ou luneta. Obtenha a relação entre as magnitudes limite do olho e do binóculo ou luneta, em concordância com a relação obtida na atividade anterior.

\section{$\sigma^{a}$ atividade: exercício teórico}

Considere uma distribuição homogênea (em um espaço euclidiano) de objetos puntiformes de mesma luminosidade, podendo ser observados até um determinado fluxo-limite $f_{0}$. Mostre que o número de objetos que podem ser observados, isto é, $\mathrm{N}(f>f 0)$, é proporcional a $f^{-3 / 2}$. Mostre que a dependência deste mesmo número de objetos observados em função da magnitude limite mo correspondente é dada por

$$
N\left(m<m_{0}\right)=N_{0} 10^{0.6 m_{0}},
$$

onde $N_{0}$ é uma constante de normalização.

Mostre ainda que a mesma relação é válida para uma distribuição homogênea de objetos de luminosidades diferentes ${ }^{7}$.

$7^{a}$ atividade: manuseio de software

Escolha uma seção do céu apresentado na carta celeste, por exemplo, na região da Via Láctea. Conte o número de estrelas apresentado nesta seção. Vá variando o valor da magnitude limite no filtro de magnitudes (por exemplo, faça 
variações de uma unidade) e repetindo a contagem do número de estrelas que aparecem na seção escolhida. Faça um gráfico de $\log N(m<m 0)$ em função de mo e compare com a relação esperada para uma distribuição homogênea de estrelas.

$8^{a}$ atividade: observações a olho nu e com instrumentos e manuseio do software

Observe uma seção do céu noturno a olho nu e conte o número de estrelas nela observadas. Observe esta mesma seção com um binóculo ou luneta e repita a contagem de estrelas. Verifique a compatibilidade das contagens com a relação obtida no exercício 6 .

\section{Solução dos exercícios e execução das atividades}

\section{$1^{a}$ atividade: manuseio do software}

Trata-se de exercício de mera familiarização com o uso do filtro de magnitudes do software. Naturalmente, espera-se a pronta constatação de que a diminuição da magnitude limite leva à presença de um número de objetos muito menor que o que aparece com o emprego de uma magnitude limite mais elevada.

\section{$2^{a}$ atividade: observação a olho nu e manuseio do software}

Consegue-se maior precisão com o emprego de uma região do céu com estrelas cujas magnitudes cubram uma gama extensa de valores. Uma sugestão é a constelação do Escorpião. Outra é a região do Cruzeiro do Sul, embora, neste caso, se possível, seria interessante abranger alguma área também da constelação do Centauro. Esta atividade foi realizada pelos autores em uma área rural, com pouca luminosidade, em 15/08/2009, e em Belo Horizonte, na noite seguinte. Em ambas as noites, não havia Lua.

No caso de Belo Horizonte, foi observado o Cruzeiro do Sul, por volta de 19 horas. Apenas três estrelas eram visíveis (exatamente Alfa, Beta e Gama Crucis), sendo que, em alguns momentos tínhamos um vislumbre de Delta Crucis. Empregando o software Redshift, em sua versão 2, descobrimos que quando colocávamos a magnitude limite em 3,5, chegávamos à condição de vermos as três estrelas. Como o filtro de magnitudes desse programa é analógico (desloca-se um ponteiro sobre uma escala, ao invés de digitar-se um número), entendemos que há alguma imprecisão no processo, mas, em primeira análise, poderíamos afirmar que essa seria a magnitude-limite naquele momento. Por outro lado, a magnitude de Delta Crucis, que parecia marcar exatamente o menor brilho (e, portanto, a maior magnitude observável) é 3,1, o que torna nossa conclusão aceitável.

No caso da área rural, foi observada a constelação do Escorpião. Foram consideradas as estrelas que compõem a figura da Constelação, excluídos os aglomerados. A contagem a olho nu chegou a 34 estrelas. $\mathrm{O}$ mesmo número foi conseguido no Redshift com magnitude limite próxima de 5,2. Embora seja 
um valor aproximado, vê-se claramente que se trata de uma magnitude possível em condições ideais, de valor 6 .

$3^{a}$ atividade: fotografia do céu noturno e manuseio do software

A foto que analisamos ${ }^{8}$ foi tratada no software Paint, encontrado na Pasta Acessórios, do Windows. Ela foi transformada em um arquivo preto e branco (Menu Imagem - Atributos - Preto e Branco), e a seguir, fizemos a inversão de cores (Menu Imagem - Inverter Cores). Para quem está acostumado a observar o céu, ela mostra, sem dúvida, a região da cauda do Escorpião, sendo Antares o objeto na parte superior da foto, ligeiramente à esquerda do centro (veja a Figura 1). Os outros objetos estão identificados na própria foto.

Figura 1
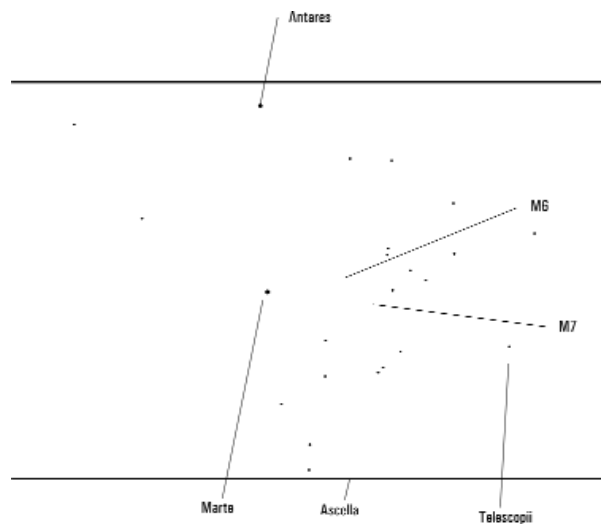

O programa de que nos valemos é o CyberSky. Por meio dele, identificada a região, concluímos que, na vertical, a foto corresponde a cerca de $33^{\circ}$, enquanto na horizontal temos cerca de $50^{\circ}$. A dimensão vertical foi descoberta pelas alturas (conforme indicadas no software) das estrelas que aparecem: no extremo superior, um pouco acima de Antares, e no meio do extremo inferior, Ascella ( $\zeta$ Sagitarii). A dimensão horizontal foi determinada fazendo-se a comparação da dimensão horizontal da foto com sua dimensão vertical, fazendo-se a partir daí um simples cálculo de regra de três. Naturalmente, isso é uma aproximação, na qual supôs-se o céu perfeitamente planificado, o que só é válido para áreas bem pequenas da abóbada celeste.

Uma das estrelas de menor brilho parece-nos ser $\delta 1$ Telescopii, apontada na figura, praticamente não visível ali, mas perceptível quando a foto é aberta no Paint do Windows. Sua magnitude é 4,96, o que nos permite situar a magnitude limite dessa foto em aproximadamente 5,0. A foto abrange regiões das constelações de Scorpius (Escorpião), Sagittarius (Sagitário), Corona Australis (Coroa Astral), Telescopium (Telescópio), Ara (Altar), Ophiucus (Ofiúco), Scutum (Escudo), e Serpens (Serpente). 


\section{$4^{a}$ atividade: exercício teórico}

Seja io a resposta do primeiro sensor à estrela de menor brilho, ou maior magnitude $(m 0)$, que é a menor resposta que esse sensor dá, correspondente a um fluxo fi. A menor resposta do segundo sensor também será $i$, mas corresponderá a um fluxo $f 2$ diferente. Com a hipótese de que $i \propto$ (área do espelho $\mathrm{x}$ fluxo) e expressando essa menor resposta do primeiro e do segundo sensor, teremos:

$$
\pi \frac{D_{0}^{2}}{4} f_{1}=\pi \frac{\left(\alpha D_{0}\right)^{2}}{4} f_{2} \Rightarrow \frac{f_{1}}{f_{2}}=\alpha^{2}
$$

Portanto, a estrela de maior magnitude captada pelo segundo sensor terá brilho $f_{2}=f_{1} / \alpha^{2}$, e a magnitude $m 2$ dessa estrela será, por (1),

$$
m_{2}=m_{0}+2,5 \log _{10} \alpha^{2}=m_{0}+5 \log _{10} \alpha
$$

Assim, a magnitude limite do segundo sensor é:

$$
m_{0}+5 \log _{10} \alpha
$$

\section{$5^{a}$ atividade: caracterização do instrumento de observação}

$\mathrm{O}$ diâmetro da pupila, quando se está observando o céu à noite, em área escura, após adaptação à falta de luz, atinge seu valor máximo, que é cerca de sete milímetros, variando um pouco de uma pessoa para outra. Esse valor cai com a idade, sendo cerca de $6 \mathrm{~mm}$ na casa dos trinta anos, diminuindo para 5 $\mathrm{mm}$ e até $4,5 \mathrm{~mm}$ após os quarenta anos.

Já o binóculo empregado em nossas observações é um instrumento 7 x 50, ou seja, tem abertura (diâmetro da objetiva) igual a $50 \mathrm{~mm}$ e proporciona aumento de sete vezes. Como o "sensor" nesta atividade será o mesmo (a retina do observador), poderemos nos valer da expressão obtida na atividade anterior, conforme solicitado em seu enunciado. Aqui, estamos fazendo a hipótese de que toda a luz que emerge pela ocular do binóculo é captada pelo olho do observador, ou seja, a pupila de saída é adequada ao diâmetro da pupila do observador. $\mathrm{O}$ valor desta grandeza (diâmetro do feixe de luz na saída da ocular) é obtido dividindo-se a abertura do binóculo pelo seu aumento. No caso do binóculo 7 x 50 , este valor é $50 / 7$, ou seja, $7 \mathrm{~mm}$, em primeira aproximação. Utilizando um diâmetro de $7 \mathrm{~mm}$ para a pupila, nossa hipótese de aproveitamento total da luz estará satisfeita. Daí, $\alpha=50 / 7=7,14$, e a relação entre as magnitudes limites a olho nu (mo) e com o binóculo (m2) será

$$
m_{2}=m_{0}+5 \log _{10} \alpha=m_{0}+5 \log _{10} 7,14=m_{0}+4,27
$$


Portanto, se, por exemplo, em determinada noite a magnitude limite para esse observador é, a olho nu, 4, com o binóculo será aproximadamente 8,3.

Note-se que essa pequena discussão mostra que binóculos 10 x 50 podem até ser mais adequados do que os 7 x 50, especialmente para observadores de idade madura. É que a saída de pupila daqueles, $5 \mathrm{~mm}$, corresponde melhor ao diâmetro da pupila desses observadores, havendo menor possibilidade de perda de aproveitamento da luz que sai pela ocular e adentra o olho do observador. Caso haja perda, o ganho na magnitude limite com o uso do binóculo será menor do que aquele que calculamos neste exercício.

\section{$\sigma^{a}$ atividade: exercício teórico}

Se os objetos têm a mesma luminosidade $L$, o número de objetos observados será limitado pela distância. Seja $r$ a distância tal que o fluxo observado seja $f_{0}$; então, $f_{0}=L /\left(4 \pi r^{2}\right)$. Com a distribuição homogênea, o número $\mathrm{N}\left(f>f_{0}\right)$ de objetos observados é dado pela densidade de objetos multiplicada pelo volume da esfera de raio ro. Logo,

$$
N\left(f>f_{0}\right) \propto \frac{4}{3} \pi r_{0}^{3} \propto r_{0}^{3} .
$$

Por outro lado,

$$
r_{0}^{2}=\frac{L}{4 \pi} \frac{1}{f_{0}} \propto f_{0}^{-1} \Rightarrow r_{0} \propto f_{0}^{-1 / 2} .
$$

Reunindo as duas relações, concluímos que, o que dá

$$
N\left(f>f_{0}\right) \propto f_{0}^{-3 / 2} .
$$

Para obter a outra relação solicitada nesta atividade, admitamos que ao fluxo $f_{1}$ corresponda uma magnitude $m$. De (1), trocando os índices 2 e 1 , respectivamente, por 1 e 0 , para ajuste de notação, vem que

$$
\frac{m_{1}-m_{0}}{2,5}=\log _{10}\left(\frac{f_{0}}{f_{1}}\right) \Rightarrow \frac{f_{0}}{f_{1}}=10^{0,4\left(m_{1}-m_{0}\right)} \Rightarrow f_{0}=\left(f_{1} 10^{0,4 m_{1}}\right) 10^{-0,4 m_{0}}
$$

Agora, aplicando (4), e considerando o termo entre parênteses uma constante (a magnitude $f_{1}$ e sua correspondente magnitude $m 1$ ajudarão a compor a constante de proporcionalidade), virá que

$$
N\left(m<m_{0}\right)=N\left(f>f_{0}\right) \quad \propto \quad f_{0}^{-3 / 2} \propto\left(10^{-0,4 m_{0}}\right)^{-3 / 2}=10^{0,6 m_{0}}
$$


Chamando a constante de proporcionalidade de $N_{0}$, chegamos à expressão solicitada, (3):

$$
N\left(m<m_{0}\right)=N_{0} 10^{0.6 m_{0}}
$$

Para comprovar que as relações (3) e (4) valem para uma distribuição uniforme de objetos de luminosidade diferentes, seja $n(L)$ a densidade, uniforme, de objetos de luminosidade L. Então, retomando o raciocínio do primeiro parágrafo desta unidade, para um dado $L$, o número de objetos observados com $f$ maior que fo é

$$
N_{L}\left(f>f_{0}\right)=n(L) \frac{4}{3} \pi r_{0}^{3}=n(L) \frac{4}{3} \pi\left(\frac{L}{4 \pi f_{0}}\right)^{3 / 2}=n(L) L^{3 / 2} \frac{(4 \pi)^{-1 / 2}}{3} f_{0}^{-3 / 2}=B n(L) L^{3 / 2} f_{0}^{-3 / 2},
$$

onde $B$ representa os fatores numéricos constantes. Para considerarmos diferentes luminosidades $L$, bastará considerarmos $N_{L}$ função de $L$, donde o acréscimo $d N_{L}$ quando a luminosidade sofrer um acréscimo $d L$ será

$$
d N_{L}=B n(L) L^{3 / 2} f_{0}^{-3 / 2} d L
$$

e daí integrarmos a expressão acima para todos os valores possíveis de $L$, obtendo-se

$$
N\left(f>f_{0}\right)=B\left[\int_{0}^{\infty} n(L) L^{3 / 2} d L\right] f_{0}^{-3 / 2} .
$$

Como a integral tem um valor fixo, próprio da nossa galáxia, ou de todo o universo, se tratar-se de contagem de galáxias, fica claro que novamente prevalece a expressão (4).

\section{$7^{a}$ atividade: manuseio de software}

Foi utilizado o software Cartes du ciel, já mencionado. Empregamos apenas catálogos de estrelas, e os aglomerados apresentados foram ignorados.

Inicialmente, decidimos examinar a região do centro da Via Láctea, cujas coordenadas são ${ }^{9} \alpha=17 \mathrm{~h} 42 \mathrm{~min}$ e $\delta=-28^{\circ} 55^{\prime}$. O software mencionado exibe uma grade de coordenadas equatoriais que nos permitiu selecionar a região definida pelos limites $17 \mathrm{~h} 40 \mathrm{~min}<\alpha<17 \mathrm{~h} 50 \mathrm{~min}$ e $-30^{\circ} 00^{\prime}<\delta<-28^{\circ} 00^{\prime}$, que contém o centro referido. A primeira magnitude limite em que surgiram estrelas foi $m=8$. As imagens obtidas são mostradas a seguir, acompanhadas da tabela com os dados obtidos. 
Figura 2

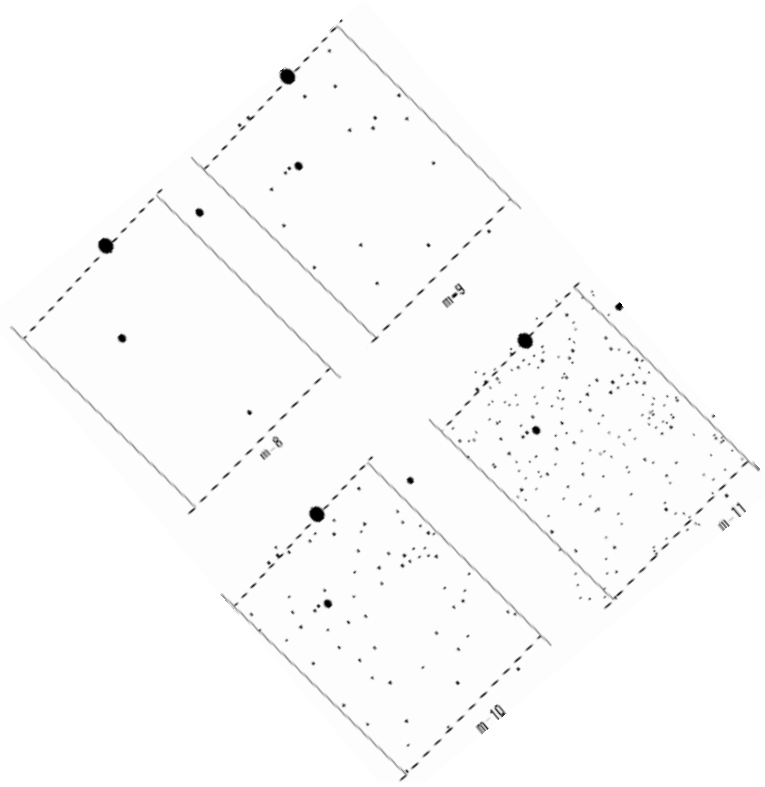

Tabela 1

\begin{tabular}{c|c}
$\mathbf{m}_{\mathbf{0}}$ & $\mathbf{N}$ \\
\hline 8 & 3 \\
\hline 9 & 19 \\
\hline 10 & 66 \\
\hline 11 & 186
\end{tabular}

O gráfico obtido está exposto abaixo.

Figura 3

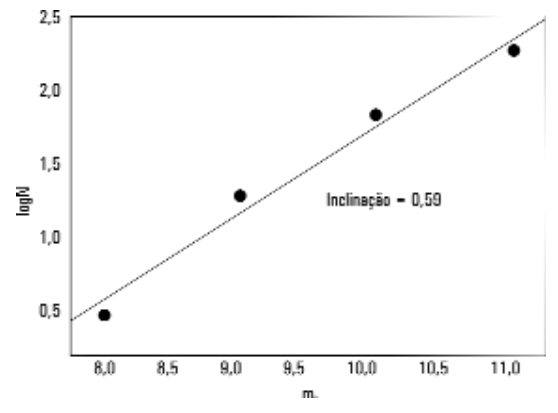

O valor da inclinação está notavelmente próximo ao valor esperado $(0,60)$.

O mesmo procedimento foi adotado para a região diametralmente 
oposta, denominada anticentro, cujas coordenadas ${ }^{10}$ são $\alpha=6$ h17min e $\delta=$ $+22^{\circ} 30^{\prime}$. A região escolhida para contagem foi a delimitada por 6h10min $<\alpha<$ 6 h20min e $+20^{\circ} 00^{\prime}<\delta<+22^{\circ} 00^{\prime}$. Desta vez, já em magnitude 7 observamos estrelas, e os dados e o gráfico obtidos estão representados no gráfico e na tabela que se seguem.

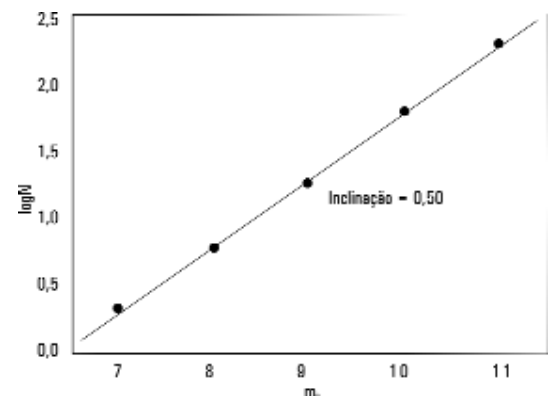

Figura 4

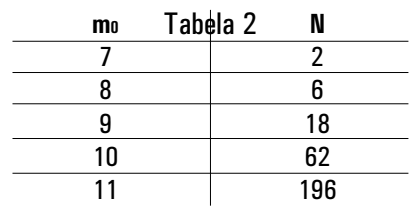

Agora, observamos um valor abaixo do esperado para o coeficiente angular, denotando uma provável queda de densidade dos objetos ao longo da linha de visada, associada à distribuição inomogênea de objetos, como se demonstrará no Apêndice.

Também foi feita uma contagem em direção perpendicular ao disco, apontando para $b$ (latitude galáctica) $=90^{\circ}$, que corresponde às coordenadas equatoriais $\alpha=0 \mathrm{~h} 49 \min$ e $\delta=+27^{\circ} 24^{\prime}$. A região escolhida no software foi 0h40min $<\alpha<0$ h50min e $+26^{\circ} 00^{\prime}<\delta<+28^{\circ} 00^{\prime}$. Os dados foram:

\begin{tabular}{l|l|lll|l|l}
$\mathbf{m}_{0}$ & 8 & 9 & Tabela 3 & 10 & 11 & 12 \\
\hline $\mathbf{N}$ & 3 & 11 & & 27 & 69 & 148
\end{tabular}

A inclinação obtida no gráfico $\log N \times m$ foi 0,42 , de novo menor que 0,6, mas compatível, como o caso anterior, com a hipótese de densidade decrescente mencionada acima, situação esperada tanto quando nos afastamos do centro (indo ao anticentro) quanto quando nos afastamos do disco galáctico 
(seguindo na linha $b=90^{\circ}$ ).

$8^{a}$ atividade: observações a olho nu e com instrumentos e manuseio do software

A primeira observação ocorreu em área rural, no dia 15 de agosto, por volta de 20 horas. A região escolhida foi a cauda do Escorpião, numa área delimitada por cinco estrelas, sendo três delas quase perfeitamente alinhadas ( $\lambda$ Scorpii, $\kappa$ (kapa) Scorpii e 11 (iota 1) Scorpii) que, com $v$ e $\theta$ Scorpii, formam, graças ao referido alinhamento, um quadrilátero. Esse quadrilátero foi usado como moldura para contagem de estrelas no seu interior. Percebia-se dentro desse quadrilátero apenas uma estrela. Tal região pode ser vista integralmente no campo de $7,1^{\circ}$ do binóculo 7 x 50 empregado. O campo se apresenta riquíssimo, inclusive com a presença de alguns aglomerados, que foram desprezados. A contagem é difícil, porque ao contrário do que se vê a olho nu (onde podemos praticamente apontar cada objeto) ou do que está na tela de um computador (onde podemos ir cobrindo ou marcando os objetos contados), no campo binocular não podemos marcar os objetos contados. Assim, a todo momento sente-se ter "perdido" a contagem, sendo necessário retomar o processo. No caso, depois de várias tentativas, concluímos que dentro do referido quadrilátero havia 32 estrelas. Usando o Cartes $d u$ Ciel, verificamos que esse número corresponde à magnitude limite 8,6. De acordo com a $5^{a}$ atividade, isso corresponderia a uma magnitude limite 4,3 para essa observação a olho nu. Voltando ao software, essa magnitude mostraria exatamente os cinco objetos mencionados, mais uma única estrela, resultado perfeitamente compatível com a contagem a olho nu. De acordo ainda com a $6^{\mathrm{a}}$ atividade, empregando a expressão (3), vê-se que isso corresponderia a um coeficiente angular 0,35 , valor bem abaixo do esperado para uma distribuição homogênea $(0,6)$, porém próximo a um dos valores encontrados na $7^{\mathrm{a}}$ Atividade $(0,42)$ a partir de contagens no software Cartes du Ciel.

\section{Conclusões}

Apresentamos neste trabalho uma sequência de exercícios e atividades, intercalando exercícios teóricos, uso de softwares e observações, dentro da proposta de exploração multicontextual de Ausubel, acompanhada de um exemplo de sua execução. Esses exercícios estão centrados na contagem de estrelas.

$\mathrm{Na}$ parte observacional, o importante é que se percebe um aumento significativo do número de objetos ao se usar um instrumento ótico de observação, e isso está relacionado ao aumento no poder de captação de luz. Uma relação entre as contagens a olho nu e com o auxílio do instrumento pode ser prevista, mas é dependente da distribuição espacial das estrelas.

Essa sequência de exercícios auxilia, portanto:

- no aprofundamento da compreensão do conceito de magnitude limite;

- na familiarização com noções de observação do céu;

- no treinamento do aluno no uso inteligente de softwares do tipo car- 
tas celestes;

- na formação do espírito de pesquisa científica, na medida em que, diante de resultados inesperados, buscam-se hipóteses que os justifiquem, as quais podem ser submetidas a novos testes;

- em uma abertura para uma discussão acerca da distribuição de objetos astronômicos, seja na Galáxia ou, em maior escala, no Universo.

A nossa prática no ensino dos conteúdos de Astronomia aqui abordados corrobora a constatação de Ausubel de que a execução multicontextual de exercícios promove a assimilação de conteúdos. A sequência de exercícios proposta, portanto, pode ser considerada constituindo os organizadores prévios, na terminologia de sua teoria. Nesse quadro teórico, em linhas gerais, o conhecimento decorre da interação entre ideias carregadas de significado (em razão de seu enraizamento em uma cultura), que já façam parte do "saber" de que o aprendiz dispõe, e o "mecanismo mental" desse aprendiz, que o capacita a adquirir e reter conhecimentos.

Julgamos que essa interação é bem conduzida neste trabalho, considerando a multiplicidade de abordagens, todas girando em torno de um conceito, que constitui o objetivo da assimilação. Imagens do céu e o uso do computador fazem parte da cultura científica na qual o aluno está imerso, enquanto os conceitos mais abstratos, necessários para organizar esse conhecimento, dentre eles o de magnitude, aguardam a vez de integrarem as aptidões de um profissional da área, seja pesquisador, seja professor.

\section{Apêndice}

Foi observada uma tendência à obtenção, nos gráficos de $\log N$ x mo da $7^{a}$ Atividade, de valores da inclinação menores que o esperado (que era 0,6, conforme a expressão (3). A hipótese mais razoável foi a de que não estaríamos diante de uma distribuição uniforme de objetos, e sim diante de uma distribuição cuja densidade cairia com a distância. Neste Apêndice, mostraremos que, para uma forma particular de queda da densidade com a distância, o resultado seria mesmo uma inclinação menor naqueles gráficos.

Vamos supor que a diminuição da densidade com o aumento de $r$

$$
n_{L}=n_{0 L}\left(\frac{R_{L}}{r_{0}}\right)^{x} \quad(x>0)
$$

possa ser aproximada por

, sendo e $r$ constantes, tudo isso para objetos de luminosidade cons-

$$
N_{L}=4 \pi \int_{0}^{r_{0}} n_{L}\left(r_{0}\right) r_{0}^{2} d r_{0}=4 \pi n_{0 L} R_{L}^{x} \int_{0}^{r_{0}} r_{0}^{2-x} d r_{0}=\frac{4 \pi n_{0 L} R_{L}^{x}}{3-x} r_{0}^{3-x} .
$$


tante $L$. Seguindo raciocínio similar àquele exposto na $6^{\text {a }}$ Atividade, teremos:

$$
r_{0}=\left(\frac{L}{4 \pi}\right)^{1 / 2} f_{0}^{-1 / 2}
$$

Mas, de (2),

$$
N_{L}=\frac{4 \pi n_{0 L} R_{L}^{x}}{3-x}\left(\frac{L}{4 \pi}\right)^{\frac{3-x}{2}} f_{0}^{(-3+x) / 2}
$$

Logo,

Se considerarmos diferentes luminosidades, $N_{L}$ será substituído por $d N^{L}$. Na nossa hipótese inicial sobre $n_{L}$, trocaremos por $\mathrm{dN}_{\mathrm{L}}$. Então a expressão

$$
N\left(f>f_{0}\right)=\int_{L=0}^{L=\infty} \frac{4 \pi \rho_{0 L} R_{L}{ }^{x}}{3-x}\left(\frac{L}{4 \pi}\right)^{\frac{3-x}{2}} f_{0}^{(-3+x) / 2} d L .
$$

acima, para $d N_{L}$, será integrada sobre todos os valores de $L$ para obtermos:

Supondo $x$ independente de $L$, por simplicidade do modelo, podere-

$$
N\left(f>f_{0}\right)=A f_{0}^{(-3+x) / 2}
$$

mos concluir que

$$
f_{0} \propto 10^{-0,4 m_{0}}
$$

, $\operatorname{com} A$ constante. Mas como

(cf. desenvolvimento da $6^{\mathrm{a}}$ atividade), poderemos reescrever a expressão acima

$$
N\left(m<m_{0}\right)=c\left(10^{-0,4 m_{0}}\right)^{(-3+x) / 2}=c \cdot 10^{\left(-0,4 m_{0}\right)(-3+x) / 2}=c \cdot 10^{(0,6-0,2 x) m_{0}}
$$

como

$$
\log N\left(m<m_{0}\right)=\log c+(0,6-0,2 x) m_{0}
$$

Daí,

Portanto, em um gráfico de $\log N(m<m 0)$ x $m 0$, a inclinação será $0,6-0,2 x$, ou seja, ela é menor que 0,6 para $x>0$. 


\section{NOTAS}

1 Oliveira Filho \& Oliveira Saraiva, 2000, p. 168.

2 Cf. http://www.ecuadors.net/astronomy/astroprog.htm (consultado em 12/08/2009).

3 Cf. http://www.astrosurf.com/astropc/cartes/index.html (consultado em 12/08/2009).

${ }^{4}$ Cf. http://www.stellarium.org/pt/ (consultado em 12/08/2009).

5 Cf. http://www.redshift7.com/ (consultado em 12/08/2009).

6 Cf. http://www.cybersky.com/ (consultado em 12/08/2009).

7 A relação (3) foi empregada para testar a homogeneidade do Universo, proposta por Kapteyn no início do século XX [5]. O universo de Kapteyn abrangia somente uma parte da Via Láctea, centrada no Sol. A contagem de estrelas em número inferior à prevista por esta relação para grandes magnitudes, isto é, para estrelas mais fracas, sugeria a presença de uma borda para o universo de Kapteyn. Mais recentemente, esta mesma relação, agora aplicada a galáxias, também foi usada para testar a homogeneidade do Universo em grande escala. Esta relação aplica-se aproximadamente às galáxias com magnitude aparente na faixa de 14 a 20 . Abaixo da magnitude 14 , o desvio deve-se à aglomeração local de galáxias, enquanto o desvio para as galáxias de magnitude superior a 20 deve-se provavelmente à sua evolução ou desvios em relação à geometria euclidiana do universo, pronunciada em distâncias maiores [6].

8 Gentilmente cedida pelo Prof. Sérgio Luís Araújo Vieira.

9 Oliveira Filho \& Oliveira Saraiva, 2000, p. 441.

10 Cf. http://astronomy.swin.edu.au/cms/astro/cosmos/G/Galactic+Anti-Centre.

\section{REFERÊNCIAS BIBLIOGRÁFICAS}

AUSUBEL, David P. Aquisição e retenção de conhecimentos: uma perspectiva cognitiva. Lisboa: Plátano Edições Técnicas, 2003. 243p.

BERNARDES, T. O. et al. Abordando o ensino de óptica através da construção de telescópios. Revista Brasileira de Ensino de Física. São Paulo, v. 28, n. 3, p. 391-396, 2000.

OLIVEIRA FILHO, Kepler de Souza \& OLIVEIRA SARAIVA, Maria de Fátima. Astronomia e Astrofísica. Porto Alegre: Ed. Universidade/UFRGS, 2000. 441p.

PEEBLES, P. J. E. The Large-Scale Structure of the Universe. Princeton University Press, 1980.

SOUZA, Ronaldo E. de. Introdução à Cosmologia. São Paulo: Edusp, 2004. 315p.

WIKIPEDIA. Disponível em >http://en.wikipedia.org/wiki/Weber-Fechner_law > Acesso em 06 set. 2009. 
